\title{
Enantiomer Separation of Mandelates and Their Analogs on Cyclodextrin Derivative Chiral Stationary Phases by Capillary GC
}

\author{
Meng-Yan NIE, ${ }^{\dagger}$ Liang-Mo ZHOU, Qing-Hai WANG, and Dao-Qian ZHU \\ Dalian Institute of Chemical Physics, Chinese Academy of Sciences, Dalian 116012, P. R. China
}

\begin{abstract}
Enantiomer separation of mandelates and their analogs, which are important intermediates in asymmetric synthetic and pharmaceutical chemistry, was investigated by capillary gas chromatography using different cyclodextrin derivative chiral stationary phases (CD CSPs). The used cyclodextrin derivatives included permethylated $\beta$-CD (PMBCD), permethylated $\gamma$-CD, heptakis(2,6-di- $O$-butyl-3- $O$-butyryl)- $\beta$-CD, heptakis(2,6-di- $O$-pentyl-3- $O$-acetyl)- $\beta$-CD and heptakis(2,6-di- $O$-nonyl-3- $O$-trifluoroacetyl)- $\beta$-CD (DNTBCD), respectively. Among all the CSPs used, PMBCD and DNTBCD exhibited the broadest and best enantioselectivity for all the racemates investigated. Some thermodynamic parameters were evaluated and an enthalpy-entropy compensation effect was observed in enantiomer separation processes of mandelates and their analogs. Based on thermodynamic data and molecular mechanics calculations, the chiral recognition mechanism of mandelate derivatives on CD CSPs is discussed.
\end{abstract}

(Received March 21, 2001; Accepted July 16, 2001)

\section{Introduction}

After the introduction of modified cyclodextrins (CDs) as a new type of chiral stationary phase (CSP), great progress has been made in enantioselective gas chromatography (GC); the research has raised many questions about the mechanisms of chiral recognition and intermolecular interactions. ${ }^{1,2}$ On CD CSPs, the enantiomers of a wide variety of structurally diverse racemates have been resolved. A number of chiral recognition mechanisms have been established depending on the particular chiral guest and cyclodextrin derivative under investigation. ${ }^{1-4}$ However, a reasonable model is still lacking to explain GC enantiomer separation on cyclodextrin based CSPs.

Optically pure $\alpha$-hydroxyacid derivatives are of interest as intermediates for the synthesis of a wide variety of products such as amino acids, angiotensin converting enzyme (ACE) inhibitors or coenzyme A..$^{5-7}$ The determination of enantiomeric purity for these $\alpha$-hydroxyacid derivatives is especially important in the study of asymmetric synthesis and quality monitoring of drug production. On pentylated $\beta$-CD and $\gamma-\mathrm{CD}$ CSPs, the enantiomers of methyl mandelate and methyl 3phenyllactate were resolved. ${ }^{8-10}$ On permethylated $\beta$-CD CSP, we achieved GC chiral separation of ethyl 2-hydroxy-4phenylbutyrate. ${ }^{11}$ In this contribution, GC enantiomeric separation of mandelates and their analogs was further investigated on different cyclodextrin derivative CSPs. Based on thermodynamic parameters and molecular modeling results, the mechanisms of enantiomer separation of mandelates and their analogs on CD CSPs are discussed.

† To whom correspondence should be addressed.

E-mail: mynie105@hotmail.com

M. Y. N. present address: Department of Chemistry, University of Reading, Whiteknights, Reading RG6 6AH, UK.

\section{Experimental}

\section{Chemicals}

Permethylated $\beta$-CD (PMBCD), permethylated $\gamma$-CD (PMGCD), $\quad$ heptakis $(2,6$-di- $O$-butyl-3- $O$-butyryl)- $\beta$-CD (DBBBCD), heptakis(2,6-di- $O$-pentyl-3- $O$-acetyl)- $\beta$-CD (DPABCD) and heptakis(2,6-di- $O$-nonyl-3- $O$-trifluoroacetyl)- $\beta$ $\mathrm{CD}$ (DNTBCD) were synthesized and purified as described earlier. ${ }^{11-13}$

Mandelic acid and $\alpha$-methoxymandelic acid were purchased from TCI (Japan). 3-Phenyllactates were prepared according to the literature method. ${ }^{14}$ Ethyl 2-hydroxy-4-phenylbutyrate is the product of asymmetric hydrogenation of ethyl pyruvate on cinchonidine-modified $\mathrm{Pt} / \mathrm{Al}_{2} \mathrm{O}_{3}$ catalyst, ${ }^{7}$ supplied by the State Key Laboratory of Catalysis (China). All other reagents were commercially available.

All the $\alpha$-hydroxycarboxylic acids were esterified with methanol or ethanol and $98 \%$ sulfuric acid, according to a previously published procedure. ${ }^{14}$

\section{Instruments and procedures}

Chromatographic separations were performed with a modified GC-1121 capillary gas chromatograph (Shanghai Analytical Instrument Factory, China) equipped with a flame-ionization detector. Both the injector and detector temperatures were maintained at $250^{\circ} \mathrm{C}$. With $\mathrm{H}_{2}$ as the carrier gas, all the separations were performed isothermally with a split ratio of $1: 100$.

Chiral columns were prepared as described earlier. ${ }^{13}$ Briefly, fused silica capillary tubes $(30 \mathrm{~m} \times 0.25 \mathrm{~mm}$ i.d. $)$ deactivated using PEG-20M were coated statically with mixture solutions of the $\mathrm{CD}$ derivatives and polysiloxane diluents dissolved in $\mathrm{CH}_{2} \mathrm{Cl}_{2}$, and then conditioned at $190^{\circ} \mathrm{C}$ for over $4 \mathrm{~h}$. 
Table 1 Chromatographic separation results of mandelates and their analogs on different CD CSPs

\begin{tabular}{|c|c|c|c|c|c|c|c|c|}
\hline \multirow{2}{*}{ CSP type } & & \multicolumn{2}{|c|}{ Mandelates } & \multicolumn{2}{|c|}{$\alpha$-Methoxymandelates } & \multicolumn{2}{|c|}{ 3-Phenyllactates } & \multirow{2}{*}{$\begin{array}{l}\text { Ethyl 2-hydroxy- } \\
\text { 4-phenylbutyrate }\end{array}$} \\
\hline & & Methyl ester & Ethyl ester & Methyl ester & Ethyl ester & Methyl ester & Ethyl ester & \\
\hline \multirow[t]{3}{*}{ PMBCD } & $k_{1}{ }^{\mathrm{a}}$ & 42.86 & 53.59 & 25.16 & 33.21 & 28.85 & 36.96 & 49.59 \\
\hline & $\alpha^{\mathrm{b}}$ & 1.048 & 1.025 & 1.019 & 1.041 & 1.090 & 1.088 & 1.077 \\
\hline & $T /^{\circ} \mathrm{C}$ & 100 & 100 & 100 & 100 & 120 & 120 & 120 \\
\hline \multirow[t]{2}{*}{$\mathrm{PMGCD}^{c}$} & $k_{1}$ & 36.74 & 52.20 & 20.90 & 28.57 & 12.53 & 17.56 & 31.45 \\
\hline & $T /{ }^{\circ} \mathrm{C}$ & 90 & 90 & 100 & 100 & 120 & 120 & 120 \\
\hline \multirow[t]{3}{*}{ DBBBCD } & $k_{1}$ & 32.26 & 39.77 & 23.63 & 31.01 & 54.95 & 74.77 & 47.17 \\
\hline & $\alpha$ & 1.021 & 1.011 & $\mathrm{NS}^{\mathrm{d}}$ & NS & 1.012 & NS & NS \\
\hline & $T /{ }^{\circ} \mathrm{C}$ & 100 & 100 & 100 & 100 & 100 & 100 & 120 \\
\hline \multirow[t]{3}{*}{ DPABCD } & $k_{1}$ & 215.1 & 74.80 & 21.75 & 28.95 & 55.64 & 76.85 & 31.63 \\
\hline & $\alpha$ & 1.011 & 1.012 & NS & NS & NS & 1.008 & NS \\
\hline & $T /{ }^{\circ} \mathrm{C}$ & 70 & 90 & 100 & 100 & 100 & 100 & 130 \\
\hline \multirow[t]{3}{*}{ DNTBCD } & $k_{1}$ & 16.19 & 21.54 & 12.87 & 17.46 & 24.87 & 36.30 & 24.47 \\
\hline & $\alpha$ & 1.071 & 1.041 & 1.024 & 1.021 & 1.021 & 1.015 & NS \\
\hline & $T /{ }^{\circ} \mathrm{C}$ & 100 & 100 & 100 & 100 & 100 & 100 & 120 \\
\hline
\end{tabular}

a. $k_{1}$ is the retention factor of the first eluted enantiomer (all $R$-enantiomers elute first). b. $\alpha$ is the separation factor. c. None of the racemates tested were resolved on PMGCD CSP. d. No enantiomeric separation was observed.

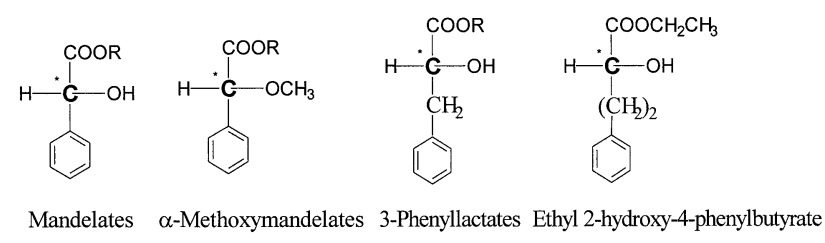

Fig. 1 Molecular structures of all the test compounds $\left(\mathrm{R}=\mathrm{CH}_{3}\right.$ and $\left.\mathrm{CH}_{2} \mathrm{CH}_{3}\right)$.

\section{Methods of molecular modeling calculations}

All the molecular mechanics calculations were carried out in vacuum on PII233 PC with MM2 force field of CS Chem3D Pro (CambridgeSoft Corp.). Non-bonded interaction cutoffs for van der Waals and dipole/dipole interactions were set as $10 \AA$ and $18 \AA$, respectively. Energy minimizations were terminated when the gradient root mean square was below $0.001 \mathrm{kcal} \mathrm{mol}^{-1}$ $\AA^{-1}$.

Permethylated $\beta$-CD structure was derived from the crystal structure of $\beta$-CD. ${ }^{15}$ The structures of both enantiomers of methyl mandelate were built using Chem3D. Before molecular simulations, PMBCD and the enantiomers were fully minimized using MM2 force field method. Similarly to the docking procedures, ${ }^{16-18}$ the inclusion complex modeling was achieved by insertion of the enantiomers into the cavity of PMBCD along an axis ( $x$-axis) perpendicular to the plane occupied by the oxygen atoms of the glycosidic linkages between glucose residues of the macrocycle. A search for minimal energy points was carried out by a stepwise penetration of the enantiomer into the cavity to a different depth and a stepwise rotation of the enantiomer around the $x$-axis within the cavity.

\section{Results and Discussion}

Enantiomer separation of mandelates and their analogs on $C D$ CSPS

The tested $\alpha$-hydroxycarboxylate racemates were methyl and ethyl mandelates, methyl and ethyl $\alpha$-methoxymandelates, methyl and ethyl 3-phenyllactates, and ethyl 2-hydroxy-4phenylbutyrate. As shown in Fig. 1, all the compounds contain a single aromatic ring, but they contain different methylene groups between the aromatic ring and the stereogenic carbon. In LC with chemical bonded $\beta$-CD stationary phases, it is generally suggested that all the resolved enantiomers containing a single aromatic ring have at least one group available for hydrogen bonding, and the aromatic ring of enantiomers resolved must be adjacent to the stereogenic carbon. ${ }^{19,20}$ On the contrary, these compounds exhibited different enantiomer separation behaviors on CD CSPs in GC. As seen in Table 1, permethylated $\beta$-CD exhibits the most extensive enantioselectivity for mandelates and their analogs, and baseline enantiomer resolution of all the tested racemates can be achieved on PMBCD CSP. It is noteworthy that both the chemical yield and the optical yield of the asymmetric hydrogenation of ethyl pyruvate can be directly and simultaneously determined from the gas chromatographic results. ${ }^{11}$ DNTBCD exhibits the best enantioselectivity for the mandelates. Except for ethyl 2-hydroxy-4-phenylbutyrate, all other racemates tested were separated on DNTBCD CSP (as shown in Fig. 2). On DBBBCD and DPABCD CSPs, enantiomeric separation of only a part of the tested racemates can be achieved. However, on permethylated $\gamma$-CD CSP, no enantiomer separation was observed for all the tested racemates. This means that in GC, chiral recognition of $\alpha$ hydroxycarboxylates containing a single aromatic ring is related to the size of cyclodextrin derivatives, and the cavity size of $\beta$ $\mathrm{CD}$ derivatives is more suitable for enantiomer separation of the racemates containing a single aromatic ring.

In addition, the data in Table 1 reveal that for the majority of the $\alpha$-hydroxycarboxylates resolved on CD CSPs, separation factors of the methyl esters are greater than those of the corresponding ethyl esters at the same temperature, which is in agreement with the trend that an increase in the carbon number of the ester group of the mandelates brings about a decrease in enantioselectivity on the $\beta$-CD bonded phase in LC. ${ }^{20}$ However, it is noteworthy that for the $\alpha$-methoxymandelates, separation factors of the methyl ester on PMBCD CSP were lower than those of the ethyl ester at the same temperature. On DPABCD CSP, all the resolved racemates exhibited the same trend as $\alpha$ methoxymandelates on PMBCD CSP.

Thermodynamic data of mandelates and their analogs on 
Table 2 Thermodynamic data of mandelates and their analogs on different CD CSPs

\begin{tabular}{|c|c|c|c|c|c|c|c|c|}
\hline \multirow{2}{*}{ CSP type } & & \multicolumn{2}{|c|}{ Mandelates } & \multicolumn{2}{|c|}{$\alpha$-Methoxymandelates } & \multicolumn{2}{|c|}{ 3-Phenyllactates } & \multirow{2}{*}{$\begin{array}{l}\text { Ethyl 2-hydroxy- } \\
\text { 4-phenylbutyrate }\end{array}$} \\
\hline & & Methyl ester & Ethyl ester & Methyl ester & Ethyl ester & Methyl ester & Ethyl ester & \\
\hline \multirow{3}{*}{ PMBCD } & $-\Delta H_{1}^{\mathrm{a}}$ & 61.83 & 63.54 & 59.71 & 61.30 & 66.48 & 67.87 & 68.14 \\
\hline & $-\Delta(\Delta H)^{\mathrm{b}}$ & 1.411 & 0.942 & 0.525 & 1.091 & 1.896 & 2.385 & 2.094 \\
\hline & $-\Delta(\Delta S)^{\mathrm{c}}$ & 3.398 & 2.331 & 1.239 & 2.552 & 4.116 & 5.349 & 4.711 \\
\hline PMGCD & $-\Delta H_{1}$ & 56.53 & 59.05 & 56.78 & 58.64 & 55.02 & 57.64 & 63.40 \\
\hline \multirow{3}{*}{ DBBBCD } & $-\Delta H_{1}$ & 63.22 & 64.83 & 59.84 & 61.34 & 64.15 & 65.57 & 70.12 \\
\hline & $-\Delta(\Delta H)$ & 0.743 & 0.390 & & & 0.747 & & \\
\hline & $-\Delta(\Delta S)$ & 1.811 & 0.961 & & & 1.904 & & \\
\hline \multirow[t]{3}{*}{ DPABCD } & $-\Delta H_{1}$ & 65.61 & 66.50 & 59.65 & 61.23 & 63.19 & 65.05 & 71.04 \\
\hline & $-\Delta(\Delta H)$ & & 0.439 & & & & 0.265 & \\
\hline & $-\Delta(\Delta S)$ & & 1.123 & & & & 0.627 & \\
\hline \multirow[t]{3}{*}{ DNTBCD } & $-\Delta H_{1}$ & 60.90 & 62.20 & 56.40 & 57.74 & 60.17 & 62.43 & 67.13 \\
\hline & $-\Delta(\Delta H)$ & 2.119 & 1.301 & 0.637 & 0.611 & 0.488 & 0.381 & \\
\hline & $-\Delta(\Delta S)$ & 5.044 & 3.122 & 1.511 & 1.450 & 1.138 & 0.899 & \\
\hline
\end{tabular}

a. $\Delta H_{1}$ is the interaction energy of the first eluted enantiomer with CD CSP, unit in $\mathrm{kJ} \mathrm{mol}^{-1}$. b. $\Delta(\Delta H)$ is the chiral discrimination energy between a pair of enantiomers resolved on CD CSPs, unit in $\mathrm{kJ} \mathrm{mol}^{-1}$. c. $\Delta(\Delta S)$ is the entropy difference between a pair of enantiomers resolved on CD CSPs, unit in $J(\mathrm{~mol} \mathrm{~K})^{-1}$.
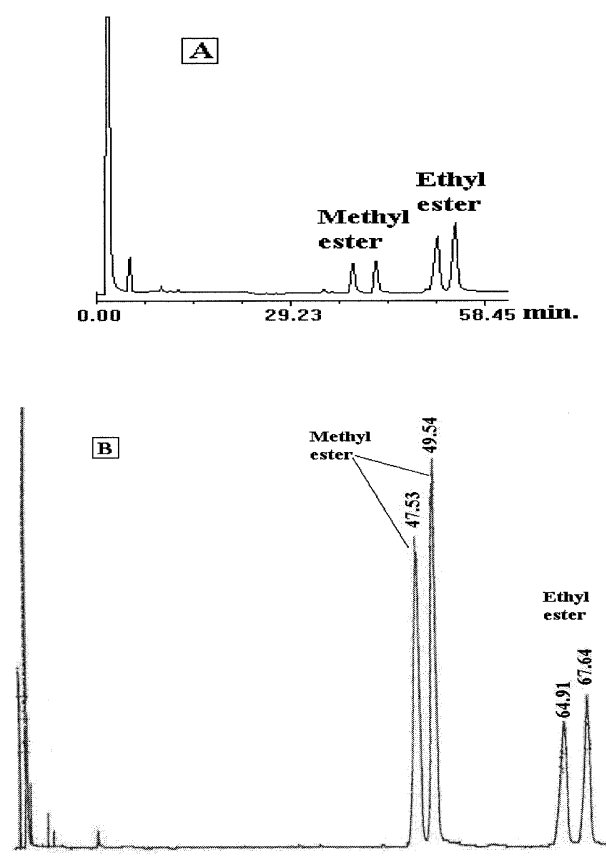

Fig. 2 Chromatograms of mandelates (A) and $\alpha$ methoxymandelates (B) separated on DNTBCD CSP in GC. Conditions: (A): column temperature, $90^{\circ} \mathrm{C}$; carrier gas flow rate, 36 $\mathrm{cm} \mathrm{s}^{-1}$; (B): column temperature, $80^{\circ} \mathrm{C}$; carrier gas flow rate, $42 \mathrm{~cm}$ $\mathrm{s}^{-1}$. The numbers above the peaks of (B) refer to the retention times in minutes.

\section{different CD CSPS}

$\Delta H$ and $\Delta(\Delta H)$ values are, respectively, direct measures of the interaction energies and the chiral discrimination interaction energies between the enantiomers and the stationary phases. These parameters can be determined from gas chromatographic retention data by use of van't Hoff equations. ${ }^{13,21}$ Thermodynamic data of mandelates and their analogs on different CD CSPs were evaluated from the van't Hoff plots with correlation coefficients higher than 0.99 , summarized in Table 2. From the table, it can be observed that the $\Delta H$ values for every enantiomer on PMGCD CSP are obviously less than the corresponding values on $\beta$-CD derivative CSPs. Due to the larger cavity size of $\gamma-\mathrm{CD}$, the enantiomers investigated cannot reach a maximum contact with PMGCD, and van der Waals interaction sites between PMGCD and the enantiomers studied are relatively few. Thus, compared with $\beta$-CD derivatives, PMGCD has relatively weaker interactions with the tested enantiomers.

In this study, all the hydroxyl groups of cyclodextrin were derivatized, and all the cyclodextrin derivatives used are no longer good hydrogen bond donors as are native CDs. Except for $\alpha$-methoxymandelates, the other enantiomers tested have good hydrogen bond donor groups (hydroxyl groups). Compared with the other enantiomers tested, $\alpha$-methoxymandelates cannot form hydrogen bonds with the used CDs, and have relatively weaker interactions with all the used CDs. In Table 2, it is obvious that $\Delta H$ values of $\alpha$-methoxymandelates on each CD CSP are less than of the other enantiomers on the corresponding CSP; this result verified that hydrogen bonding interactions are involved in the retention mechanism on $\mathrm{CD}$ CSPs. However, hydrogen bonding interaction has no significant effect on the chiral recognition mechanism of mandelates and their analogs on CD CSPs; as inferred from the data of Table 2, $\Delta(\Delta H)$ and $\Delta(\Delta S)$ values have no obvious trend between $\alpha$-methoxymandelates and the other racemates.

Enthalpy-entropy compensation effects have been successfully used to study mechanisms of various chiral recognition phenomena. ${ }^{12,13,21,22}$ As earlier suggested, in order to detect a real compensation effect, $\Delta(\Delta H)$ and $\Delta(\Delta S)$ should be measured by different methods, or statistical analysis must be performed on $\Delta(\Delta H)$ and $\Delta(\Delta S))^{12,13,21}$ For the mandelates and their analogs separated on CD CSPs, excellent correlation was observed between $\Delta(\Delta H) v s . \Delta(\Delta S)$, as shown by the equation:

$$
\Delta(\Delta H)=0.045+445.4 \Delta(\Delta S) ; S D=0.048, r=0.998, n=18
$$

Statistical analysis reveals that in the $95 \%$ confidence interval, the estimate of the slope $(\beta=445.4 \mathrm{~K})$ from $\Delta(\Delta H)$ vs. $\Delta(\Delta S)$ plot is significantly different from the harmonic mean of the experiment temperatures $\left(T_{\mathrm{hm}}=397.3 \mathrm{~K}\right)$. Therefore, it is concluded that a compensation effect exists in this data set, and that enantiomer separations of mandelates and their analogs on these CD CSPs are dominantly governed by the same chiral 

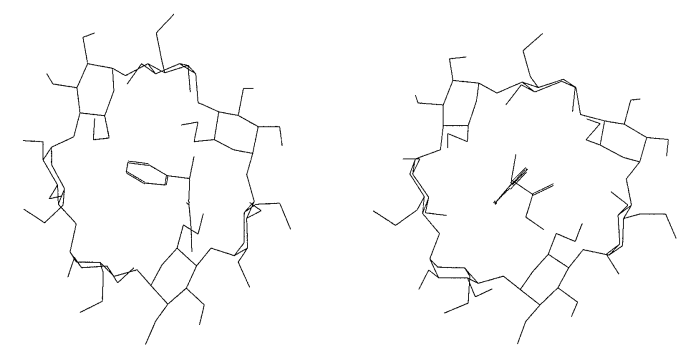

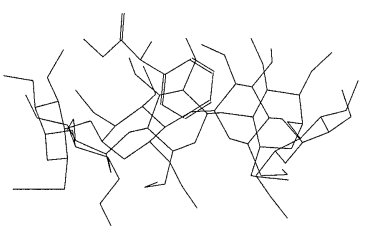

$R$-methyl mandelate-PMBCD

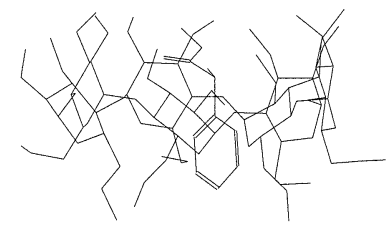

$S$-methyl mandelate-PMBCD
Fig. 3 The lowest energy conformations of $R / S$-methyl mandelatePMBCD (left/right) complexes from molecular mechanics modeling (the graphs are viewed from $x$ - and $z$-axes, respectively, and all the hydrogen atoms are hidden).

recognition mechanism. This further verified that hydrogenbonding interactions have no significant influence on the chiral recognition of mandelates and their analogs on CD CSPs, even though these interactions obviously contribute to their retentions on the CD CSPs.

As usually observed in GC, separation factors of all the tested mandelates and their analogs decrease with increasing temperature in the operating temperature ranges, and no inversion of retention order was observed. At the same time, enantiomer separations of all the tested compounds are achieved below the isoenantioselective temperature $\left(T_{\text {iso }}=\Delta(\Delta H) / \Delta(\Delta S)\right)$ (as seen from Tables 1 and 2). Thus, it can be concluded that the elution order of the enantiomers of mandelates and their analogs on CD CSPs in GC is dominantly determined by the enthalpy difference $\Delta(\Delta H) .^{23-25}$ Therefore, it is possible to predict the elution order by calculating the interaction energies of two enantiomers with cyclodextrin using molecular mechanics methods without taking the entropy change $\Delta(\Delta S)$ into account.

\section{Molecular mechanics modeling of chiral recognition between methyl mandelate and $P M B C D$}

From the molecular modeling, it is found that the preferred binding sites for methyl mandelate are in the interior of the cavity of PMBCD, and the stereocenters of the enantiomers locate near the secondary rim of PMBCD, as illustrated in Fig. 3. This indicates that the mechanisms of chiral recognition between PMBCD and methyl mandelate are involved in the formation of inclusion complexes. However, as seen in Fig. 3 , there are some differences in association modes of $R$ and $S$ enantiomers within the cavity. In the lowest energy conformations, $R$-methyl mandelate hovers over the secondary rim of PMBCD, and only the aromatic ring of the $R$-isomer was included within the cavity of PMBCD; on the contrary, $S$ methyl mandelate deeply penetrated into the cavity paralleling to the 7-fold symmetry axis of PMBCD ( $x$-axis), and was completely included within the cavity of PMBCD.

From molecular modeling, a great number of data can be produced. The values for strend, bend, torsion, van der Waals, dipole/dipole and the cross term strend-bend energies for the
Table 3 Total binding energies $(\Delta E)$, chiral discrimination energy $(\Delta \Delta E)$ and component energies for the complexes of $R / S$ methyl mandelate with PMBCD from molecular mechanics modeling calculations (units: $\mathrm{kcal} \mathrm{mol}^{-1}$ )

\begin{tabular}{lrrr}
\hline & \multicolumn{2}{c}{$R / S$-Methyl mandelate-PMBCD } \\
\cline { 2 - 4 } Energy terms & $\Delta E_{\mathrm{R}-\mathrm{CD}^{\mathrm{a}}}$ & $\Delta E_{\mathrm{S}-\mathrm{CD}}$ & $\Delta \Delta E_{\mathrm{S}-\mathrm{R}} \mathrm{b}$ \\
\hline \multirow{2}{*}{ Strend } & -0.4030 & -0.1925 & 0.2105 \\
Bend & 0.8034 & -0.5377 & -1.3411 \\
Str-bend & -0.1508 & -0.0664 & 0.0844 \\
Torsion & 0.3623 & 1.4677 & 1.1054 \\
VDW & -22.0850 & -23.8506 & -1.7656 \\
Dip/dip & -1.2779 & -0.4346 & 0.8433 \\
Total & -22.7510 & -23.6140 & -0.8630 \\
\hline
\end{tabular}

a. $\Delta E_{\mathrm{R}-/ \mathrm{S}-\mathrm{CD}}=E_{\mathrm{R}-/ \mathrm{S} \text {-enantiomer-CD complex }}-\left(E_{\mathrm{R}-/ \mathrm{S} \text {-enantiomer }}+E_{\mathrm{CD}}\right)$.

b. $\Delta \Delta E_{\mathrm{S}-\mathrm{R}}=\Delta E_{\mathrm{S}-\mathrm{CD}}-\Delta E_{\mathrm{R}-\mathrm{CD}}$.

lowest energy conformations are summarized in Table 3 along with the differences between the diastereomeric complexes. From this table, some important information can be observed. Firstly, it is obvious that the $S$-enantiomer of methyl mandelate forms more stable diastereomeric complex with PMBCD than $R$-enantiomer, which is consistent with the experimental retention order ( $R$-enantiomer interacts more weakly with PMBCD than $S$-enantiomer, and first elutes on PMBCD CSP in GC, as seen in Tables 1 and 2). Secondly, the binding energies $(\Delta E)$ of both enantiomers of methyl mandelate with PMBCD mainly come from van der Waals interaction. Thirdly, from the obvious changes of intramolecular interactions, e.g., strend, bend and torsion energies, it is inferred that the conformations of PMBCD and both enantiomers of methyl mandelate were changed during the association processes, and that the inducedfit takes place when the enantiomers are included within the cavity of PMBCD. Moreover, by analyzing all the structural parameters of free and associated PMBCD and both enantiomers, we indeed found that all the conformations of PMBCD and both enantiomers of methyl mandelate were changed during their association processes. Finally, the intramolecular interactions make few contributions to chiral discrimination energy $\left(\Delta \Delta E_{R-S}\right)$, which mainly comes from van der Waals energies. Thus, the forces mainly responsible for binding are also most responsible for chiral discrimination.

\section{Conclusions}

Among the modified cyclodextrin chiral stationary phases used, permethylated $\beta$-CD and 2,6-di- $O$-nonyl-3- $O$-trifluoroacetyl- $\beta$ $\mathrm{CD}$ exhibited best enantioselectivity for mandelates and their analogs investigated. The baseline enantiomeric resolution of all the investigated racemates can be achieved on these $\mathrm{CD}$ CSPs. It is observed that in GC, chiral recognition of mandelate derivatives is related to the cavity size of the cyclodextrin derivatives, and that $\beta$-CD derivatives are more suitable for chiral separation of $\alpha$-hydroxycarboxylates containing a single aromatic ring. Hydrogen-bonding interaction was found to contribute to the retention mechanisms of the studied mandelate derivatives on CD CSPs, but it does not contribute to the chiral recognition mechanisms of these racemates on CD CSPs. According to the statistical analysis of the thermodynamic data, an enthalpy-entropy compensation effect was observed for mandelate derivatives separated on CD CSP, which indicated that enantiomer separation of mandelates and their analogs on 
CD CSPs was dominantly governed by the same mechanism. Molecular mechanics calculations indicated that van der Waals interactions are most responsible for both association and chiral discrimination of methyl mandelate with permethylated $\beta-\mathrm{CD}$, and the mechanism of chiral recognition is involved in the formation of inclusion complexes.

\section{Acknowledgements}

Financial support from NSFC (Project No. 29775025) is gratefully acknowledged.

\section{References}

1. W. A. König, "Gas Chromatographic Enantiomer Separation with Modified Cyclodextrins", 1992, Hüthig, Heidelberg.

2. Z. Juvancz and P. Petersson, J. Microcol. Sep., 1996, 8, 99, and references cited therein.

3. N. K. Vries, B. Coussens, R. J. Meier, and G. Heemels, $H R C, \mathbf{1 9 9 2}, 15,499$.

4. K. B. Lipkowitz, Chem. Rev., 1998, 98, 1829 and references cited therein.

5. D. S. Hodgins, J. Biol. Chem., 1971, 246, 2977.

6. H. Uebach and R. Henning, Tetrahedron Lett., 1984, 25, 1143.

7. H. U. Blaser, H. P Jalett, and F. Spindler, J. Mol. Catal. A, 1996, 107,85 .

8. W. A. König, S. Lutz, M. Hagen, R. Krebber, G. Wenz, K. Baldenius, J. Ehlers, and H. Dieck, HRC, 1989, 12, 35.
9. W. A. König, R. Krebber, and P. Mischnick, $H R C, 1989$, 12,732 .

10. W. A. König, $H R C, \mathbf{1 9 9 3 , ~ 1 6 , ~} 347$.

11. M. Y. Nie, L. M. Zhou, Q. H. Wang, and D. Q. Zhu, Chin. J. Anal. Chem., 2000, 28, 1366.

12. M. Y. Nie, L. M. Zhou, X. L. Liu, Q. H. Wang, and D. Q. Zhu, Anal. Chim. Acta, 2000, 408, 279.

13. M. Y. Nie, L. M. Zhou, X. L. Liu, Q. H. Wang, and D. Q. Zhu, Chromatographia, 2000, 51, 450 .

14. F. J. Urban and B. S. Moore, J. Heterocyclic Chem., 1992, 29,431

15. C. Betzel, W. Saenger, B. E. Hingerty, and G. M. Brown, J. Am. Chem. Soc., 1984, 106, 7545.

16. D. G. Durham, Chirality, 1996, 8, 58.

17. M. Y. Nie, L. M. Zhou, Q. H. Wang, and D. Q. Zhu, Chin. Chem. Lett., 2000, 11, 347.

18. M. Y. Nie, L. M. Zhou, Q. H. Wang, and D. Q. Zhu, Acta Chim. Sinica, 2001, 59, 268.

19. D. W. Armstrong, Y. Z. Han, and S. M. Han, Anal. Chim. Acta, 1988, 208, 275.

20. K. Fujimura, M. Kitagawa, H. Takayanagi, and T. Ando, $J$. Liq. Chromatogr., 1986, 9, 607.

21. L. M. Zhou, M. Y. Nie, Q. H. Wang, D. Q. Zhu, and H. Q Shen, Chin. J. Chem., 1999, 17, 363.

22. M. V. Rekharsky and Y. Inoue, Chem. Rev., 1998, 98, 1875.

23. B. Koppenhoefer and E. Bayer, Chromatographia, 1984, 19,123

24. V. Schurig, J. Ossig, and R. Link, Angew. Chem., 1989 , 101, 192.

25. R. W. Stringham and J. A. Blackwell, Anal. Chem., 1996, 68, 2179. 